\title{
Process optimization of the extrusion through rotating container with AZ31 magnesium alloy
}

\author{
F. $\operatorname{Li}^{1 *}$, N. Bian ${ }^{1}$, Y. C. Xu ${ }^{2}$ \\ ${ }^{1}$ School of Materials Science and Engineering, Harbin University of Science and Technology, \\ Lin Yuan Street 4, Xiang Fang District, P. O. Box 317, Harbin 150040, P. R. China \\ ${ }^{2}$ National Key Laboratory of Precision Hot Processing of Metals, Harbin Institute of Technology, \\ Harbin 150001, P. R. China
}

Received 9 February 2014, received in revised form 13 May 2014, accepted 14 May 2014

\begin{abstract}
In order to study the effects of processing parameters on the extrusion through rotating container, the AZ31 magnesium alloy was employed as an example. The results by the numerical simulation show that the velocity field presents a specific characteristic vortex distribution. Compared to the conventional extrusion, the axial stress distribution difference of radial points along the die presented a tendency to decrease, and the flow uniformity of extrudate increased. The possibility of surface cracking obviously decreased. The structure refinement and load reducing can be realized by extrusion through rotating container. But the interaction effect among angular velocity of rotating container, extrusion speed and temperature had direct relation to the temperature arise and load peak. On the basis of the research of processing parameters, the limit diagram of extrusion through rotating container was established. The limit diagram provided theoretical basis for process formulation and defect prevention in the process of extrusion through rotating container.
\end{abstract}

K e y words: AZ31 magnesium alloy, extrusion through rotating container, extrusion limit, grain refinement

\section{Introduction}

With the development of technology and the increase in demand, extrusion products are becoming large in dimension, high in precision and complicated in structure. But small tonnage of equipment, low material availability and backward technology become one of bottle-necks to restrict the development of extrusion. Based on these disadvantages, a new plastic forming method was proposed by the author [1].

In the research of Mizunuma, magnesium alloy AZ31 was employed to examine the effects of temperature and speed ratio. For each die, the optimum temperature and the speed ratio required to obtain a uniform distribution of $1-2 \mu \mathrm{m}$ fine grains over the section of the billet were clarified. The billet processed by torsion extrusion had a very different crystal orientation than that of the conventionally extruded billet $[2]$.

The torsion extrusion process is characterized by rotation of a die along its axis during the extru- sion process. Several experiments were carried out to investigate the deformation characteristics and inspect microscopic structure, using aluminum and magnesium alloy billet. Due to these experiments, it can be found that there exist optimum conditions of the die and temperature, in which fracture does not occur on the billet surface and the grain size becomes very fine [3].

Nowadays, as computational power improves, manufacturing process can be considered in terms of material flow, temperature and profile speed distribution, load-stroke history, etc., by means of finite element (FE) simulation [4].

In the work performed by E. Ceretti, an FE model was tested versus experimental results obtained from an industrial plant. The accuracy of the FE model was analyzed through a dual comparison: by geometrical and physical aspects. A good agreement was found between the experiment and the simulation results [5].

The paper written by M. Ketabchi reports on an experimental and finite element analysis of a back-

*Corresponding author: tel.: +86-451-86392501; fax: +86-451-86392555;

e-mail addresses: fli@hrbust.edu.cn, hitlif@126.com 


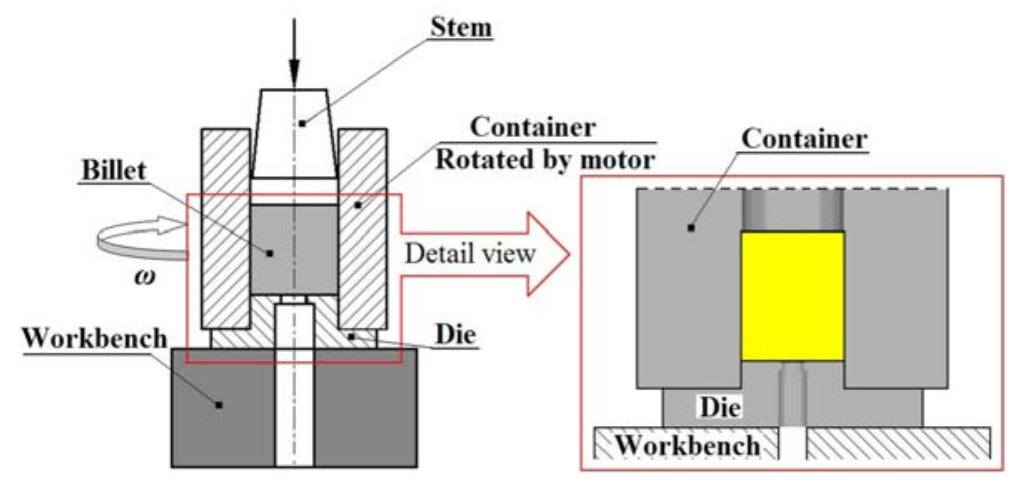

Fig. 1. Schematic illustration of extrusion through rotating container.

ward extrusion process of $7075 \mathrm{Al}$ alloy. The influence of stem speed and billet temperature on the backward extrusion process was investigated. The finite element software Deform 3D was employed for simulations. The results indicated that the effective stress/strain distributions were inhomogeneous in the deformed billet $[6]$.

Extrusion limit diagram can not only determine the reasonable work zone, but also decide the proper technological parameters. A series of experiments were carried out by Dale L. Atwell and Matthew R. Barnett in order to quantify the extrusion speed limits for key wrought magnesium alloys using a laboratory scale extrusion press in a manner that allows the effects of the different alloying additions to be assessed [7].

In the article of C. Davies and M. Barnett, extrusion behavior of wrought magnesium alloys on the rapid evaluation was examined. The work aims to establish a methodology for rapid prototyping of alloys and to assess the effects of aluminum on the behavior of AZ-series magnesium alloys [8].

Lapovok states their viewpoint of limiting extrusion speeds and ratios as a function of billet temperature. This is examined by combining hot compression test results, FEM results and extrusion trials. Hot working stress-strain curves displayed a distinct dynamic recrystallization peak. The predicted limits agree well with the occurrence of cracking in both a laboratory and a commercial extrusion trial [9].

In this paper, FE simulations were employed for the analysis of torsion extrusion process. A great deal of data can be obtained from the FE simulation. By means of these data, load variation trend, temperature field and distribution of isothermal level, velocity field and its distribution are thoroughly examined. Meanwhile, also the extrusion limit diagram and theoretical processing model are established.

\section{Research schemes}

The restraint and location structure in the extru-

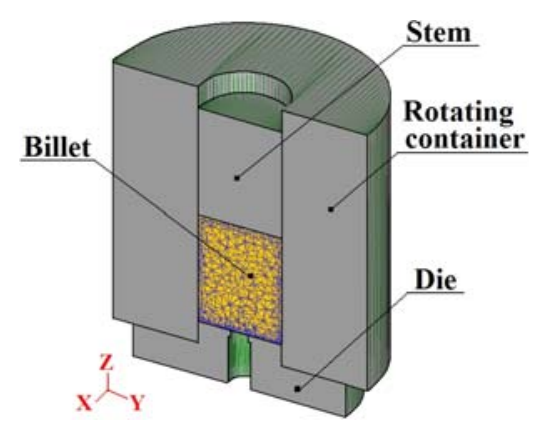

Fig. 2. FEM model.

sion process has a great impact on the stability and reliability of the system by applying a torque on the die. Therefore, the die in the extrusion process through rotating container has a flange structure. The reverse location of container can be achieved by a flange structure. The schematic illustration of extrusion through rotating container is shown in Fig. 1. The apparatus is made up of stem, container and die. The die is fixed on the workbench. The servo motor is employed for applying torque on the container.

\subsection{The finite element method}

The AZ31 magnesium alloy was used as the billet material and the H13 hot-work tool steel as the material for all the extrusion tooling in the study. Advanced simulation software Deform 3D was employed in the analysis of extrusion through rotating container. A thermo-viscoplastic material model was employed for billet and a thermo-rigid material model for extrusion tooling. The FEM model is shown in Fig. 2.

The material model neglected the elastic behavior of the billet, the physical properties parameters are given in the Table 1 . Both the billet and the extrusion tooling were dispersed by tetrahedron elements with four nodes. To improve simulation precision and efficiency and in the meantime to obtain an accurate result, local windows with a high density were applied on the area near the die. 
Table 1. Physical properties of the AZ31 billet and H13 tool steel extrusion tooling

\begin{tabular}{lcc}
\hline Property & AZ31 & H-13 tool steel \\
\hline Heat capacity $\left(\mathrm{N} \mathrm{mm}^{-2}{ }^{\circ} \mathrm{C}^{-1}\right)$ & 2.09684 at $327^{\circ} \mathrm{C}$ & 5.6 \\
Thermal conductivity $\left(\mathrm{N} \mathrm{s}^{\circ} \mathrm{C}^{-1}\right)$ & 2.27484 at $527^{\circ} \mathrm{C}$ & 96 \\
Heat transfer coefficient between tooling and billet $\left(\mathrm{N} \mathrm{s}^{-1} \mathrm{~mm}^{-1}{ }^{\circ} \mathrm{C}^{-1}\right)$ & 11 & 28.4 \\
Heat transfer coefficient between tooling/billet and air $\left(\mathrm{N} \mathrm{s}^{-1} \mathrm{~mm}^{-1}{ }^{\circ} \mathrm{C}^{-1}\right)$ & 0.02 & 11 \\
Emissivity & 0.12 & 0.02 \\
\hline
\end{tabular}
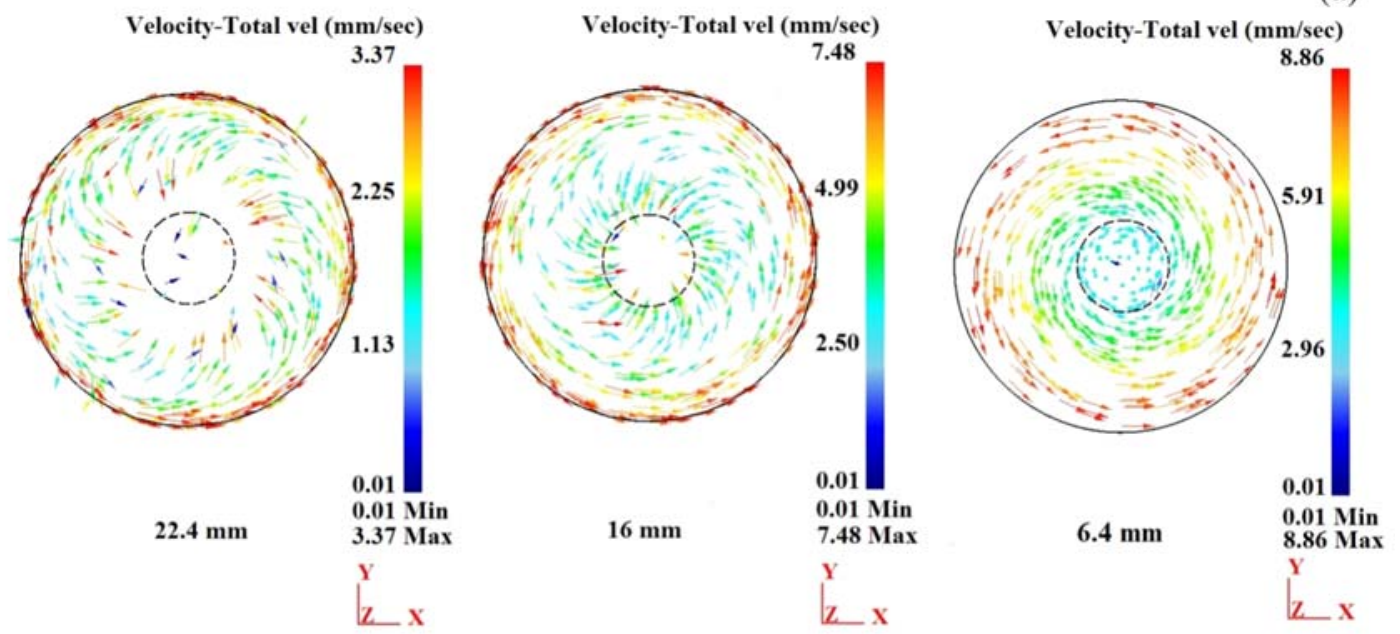

(a)

(b)
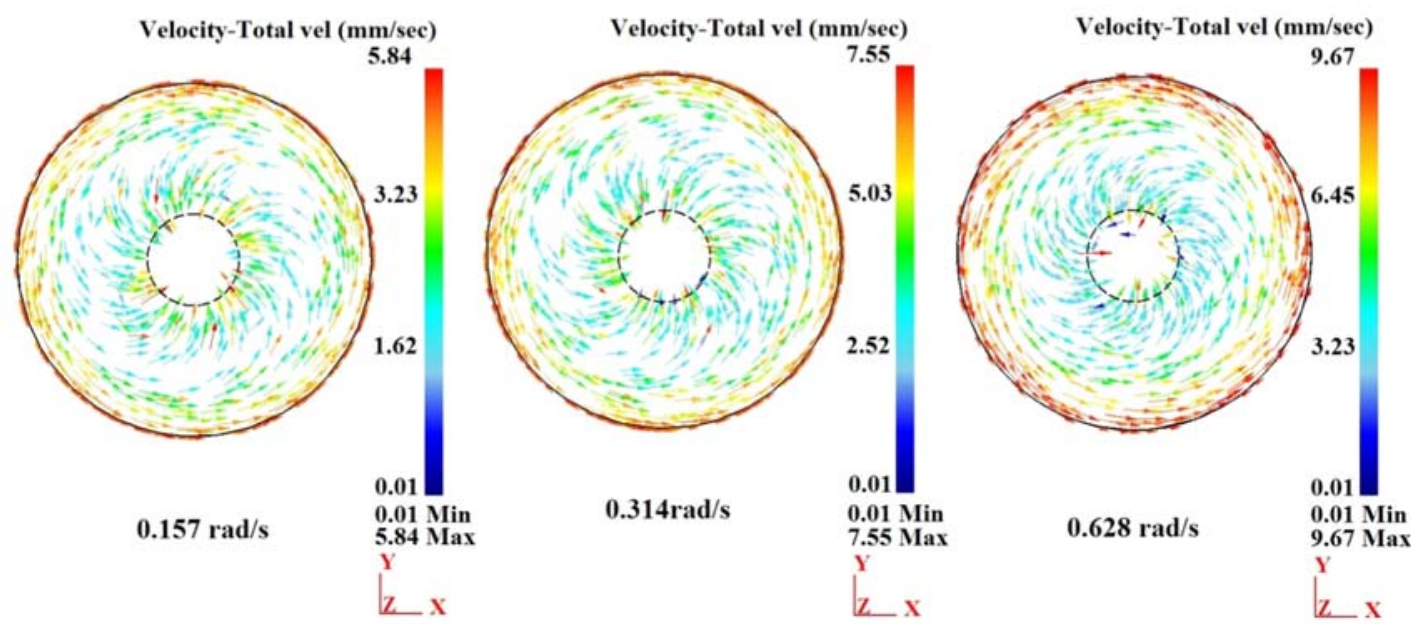

Fig. 3. Velocity field distribution under different processing parameters: section position (angular velocity of container was $0.314 \mathrm{rad} \mathrm{s}^{-1}$ ) (a), angular velocity of the container (distance from the die was $10 \mathrm{~mm}$ ) (b).

Table 2. Dimensions and initial temperatures of the billet and extrusion tooling

Billet length ( $\mathrm{mm})$

Billet diameter $(\mathrm{mm})$ 50

Container inside diameter $(\mathrm{mm})$

Container outside diameter $(\mathrm{mm})$

Billet temperature $\left({ }^{\circ} \mathrm{C}\right)$

Tooling temperature $\left({ }^{\circ} \mathrm{C}\right)$
The flow stress-strain data of AZ31 magnesium alloy were obtained from hot compression tests [10]. The processing parameters of the extrusion through rotating container in the simulation are given in Table 2 . The constant shear model defined as $f_{\mathrm{s}}=m k$ was used for the interface between the billet and the extrusion tooling ( $f_{\mathrm{s}}$ is frictional stress, $k$ is shear yield stress and $m$ is friction factor). The friction factor of 0.8 was given at the interface $[11,12]$. 
In order to examine the effect of processing parameters, other conditions and changes of the specific parameters are necessary to be compared.

\section{Results discussion and analysis}

\subsection{Metal flow behavior}

Due to the friction between the billet and container, the billet flew along the circumferential direction which was driven by the tangential component of friction after applying torque on the container. At the section perpendicular to the axial direction, the twist deformation that was derived from the friction became diminishing. The metal was extruding with vortex under the dual-loading condition - axial and circumferential. In further research, the change rule of the vortex behavior in the processing extrusion through rotating container, other conditions, the influence of section position and container rotating speed were studied, and the results are shown in Fig. 3.

The velocity field distribution at different sections of the billet is shown in Fig. 3a, when the extrusion speed was $0.314 \mathrm{~mm} \mathrm{~s}^{-1}$ and the angular velocity of container was $0.314 \mathrm{rad} \mathrm{s}^{-1}$. The vortex distribution could be seen in the extrusion through rotating container. With the section position moving down, the tendency of vortex behavior became obvious, and the flow velocity increased. Due to the higher section position, the more remarkable pinning effect yields from the friction. Therefore, most of the metal presented rigid translation with stem along the loading direction, only minority presented circumferential flow. With the section position moving down, the impact of stem that was imposing on the billet decreased. But the effect of rotating container was obvious.

The velocity field distribution with different angular velocity of container is shown in Fig. $3 \mathrm{~b}$ when the extrusion speed was $0.314 \mathrm{~mm} \mathrm{~s}^{-1}$ and the section was $10 \mathrm{~mm}$ distant from the die. With increasing of rotating speed, the degree of vortex became obvious and the velocity also increased. When the rotating speed was $0.157 \mathrm{rad} \mathrm{s}^{-1}$, the maximum velocity was $5.84 \mathrm{~mm} \mathrm{~s}^{-1}$. When the rotating speed increased to 0.314 and $0.628 \mathrm{rad} \mathrm{s}^{-1}$, the maximum velocity was increased by 29.2 and $65.6 \%$. It can be indicated from the analysis that the velocity of billet persistently increased with the increasing of rotating speed. Relative slippage appeared between the billet and the container when the rotating speed exceeded to a certain value. Therefore, increasing rotating speed promoted the flow velocity with a certain scope. But the opposite effect occurred when the rotating speed reached a certain degree.

Defects, such as bending and surface cracking, were caused by the additional stress. The axial

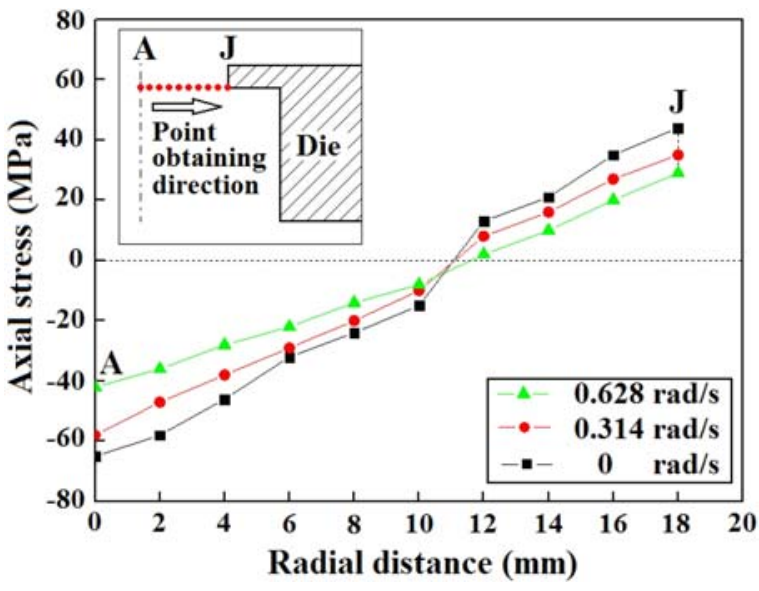

Fig. 4. Axial stress comparison of points along the radial direction with different rotating speed.

state changed after applying torque on the container. For the extrusion speed $1 \mathrm{~mm} \mathrm{~s}^{-1}$ and stem stroke $19.2 \mathrm{~mm}$, the axial stress comparison of points along the radial direction with different rotating speed is shown in Fig. 4.

It can be seen from Fig. 4 that the algebraic values of axial stress at the exit of die presented a tendency of increasing from the axis to the sidewall. When the rotating speed was $0 \mathrm{rad} \mathrm{s}^{-1}$, the tensile stress of point $J$ was $44 \mathrm{MPa}$. When the rotating speed reached 0.314 and $0.628 \mathrm{rad} \mathrm{s}^{-1}$, the stress decreased by 20 and $34 \%$. Compared with conventional extrusion, the absolute value of stress of $A$ decreased by 11 and $35 \%$, respectively.

\subsection{Twist deformation behavior}

Due to the torque applied on the container, the extrudate twisted obviously along the direction perpendicular to axial, and this is the specific characteristic of the extrusion through rotating container, as shown in Fig. 5 .

It can be seen from Fig. 5 that an incline and twist of the mesh perpendicular to the axial direction could not occur. The streamline was distributed along the axial direction. During the extrusion through rotating container, the billet flew along the axial direction and meanwhile, twist deformation occurred along the circumferential direction. It can be seen from Fig. 5b, when comparing the streamline of extrudate with conventional extrusion, the flow net perpendicular to the extrusion axis presented a vortex distribution outside-in. Surface cracking along the end-range was avoided with this distribution characteristics. The process of extrusion through rotating container enhanced the effect of shear deformation, to improve the quality of extrudate and its mechanical properties. 

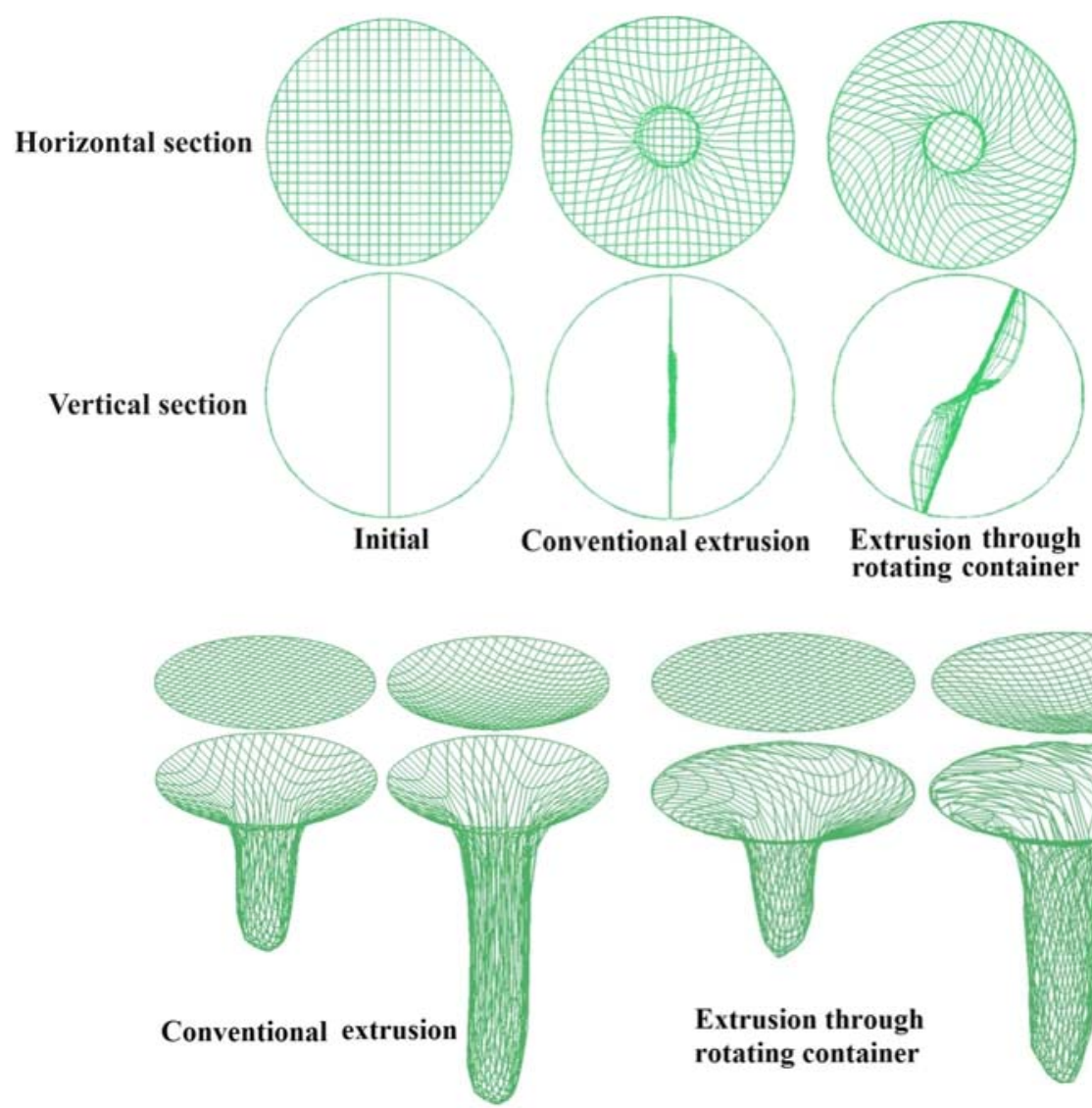

(a) rotating container

(b)

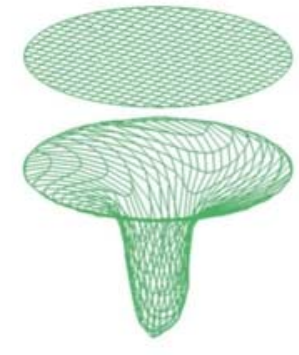

Extrusion through rotating container

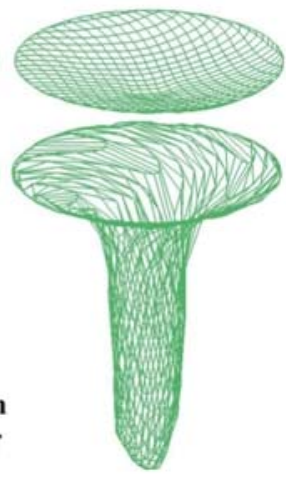

Fig. 5. Mesh comparison in the process of extrusion: section view (a), front view (b).

\subsection{Temperature rise rule}

The temperature of billet and extrusion tooling changed during the process of extrusion. With the process of extrusion, the friction heat and deformation heat had a great difference in different parts of the billet and there was a continuous change of heat transfer. Therefore, the temperature presented an obviously graded distribution. For the extrusion speed $1 \mathrm{~mm} \mathrm{~s}^{-1}$, the friction factor 0.8 and stem stroke $19.2 \mathrm{~mm}$, considering the rotating speed of $0,0.314,0.628$ and 0.942 $\operatorname{rad~} \mathrm{s}^{-1}$, the temperature distribution and its rise are shown in Fig. 6.

It can be seen from Fig. 6 a that the isothermal surface presented an increasing graded distribution during the extrusion. With increasing of rotating speed, the value of isothermal surface in the same part increased. When the rotating speed was $0 \mathrm{rad} \mathrm{s}^{-1}$, the isothermal surface at the bottom presented a hemispherical distribution as that at the center of the axis and die. The shapes of other isothermal surfaces were similar to the former one and these surfaces had almost equal interval distribution along the axial positive direction. With the increasing of rotating speed, the temperature value of isothermal surfaces on the bottom obviously increased and shapes of isothermal surfaces gradually changed to a shallow even disshaped distribution. Therefore, the temperature difference at each point in the same section obviously decreased.

In order to examine the temperature rise rule of billet, the comparison of billet maximum temperature with different rotating speed is shown in Fig. 6b. Although there was a discrepancy of rotating speed, the maximum temperatures presented a decreasing tendency after increase with the moving down of stem. From the temperature distribution, the maximum temperature of conventional extrusion was $343^{\circ} \mathrm{C}$. But when the rotating speed was $0.314 \mathrm{rad} \mathrm{s}^{-1}$, the maximum temperature was $406^{\circ} \mathrm{C}$. When the rotating speed reached 0.628 and $0.942 \mathrm{rad} \mathrm{s}^{-1}$, the maximum temperature increased by 21 and $24 \%$.

\subsection{Extrusion load}

Due to the theoretical analysis, applying torque on the container changed the direction of friction between the billet and the container. There was only a component of friction required to be overcome in the process of extrusion. Therefore, the effect of decreasing load was realized. For extrusion speed $1 \mathrm{~mm} \mathrm{~s}^{-1}$ and the friction factor 0.8 , the extrusion load comparison with 
(a)
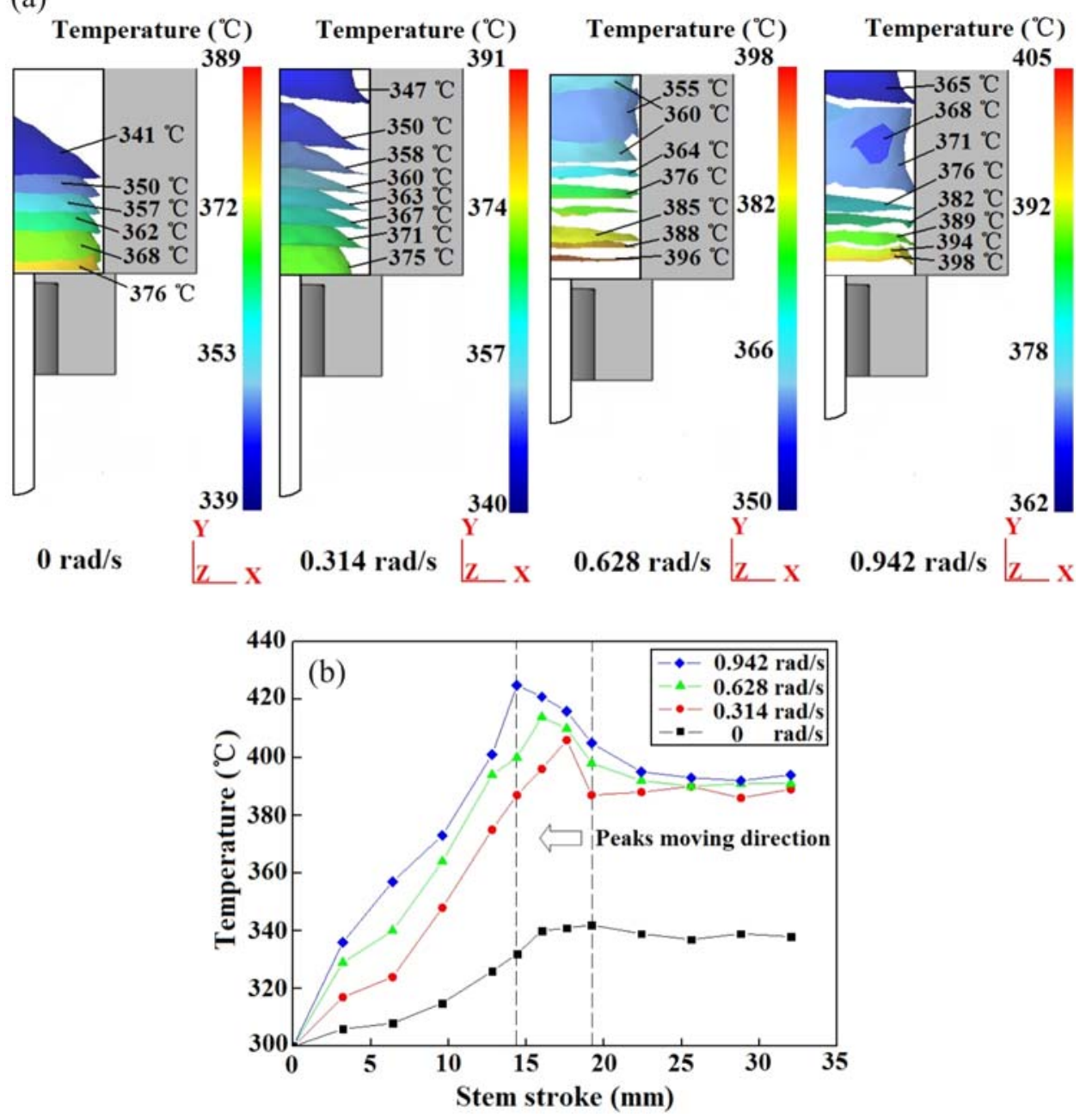

Fig. 6. Temperature rise comparison with different rotating speed: isothermal surface (a), maximum temperature (b).
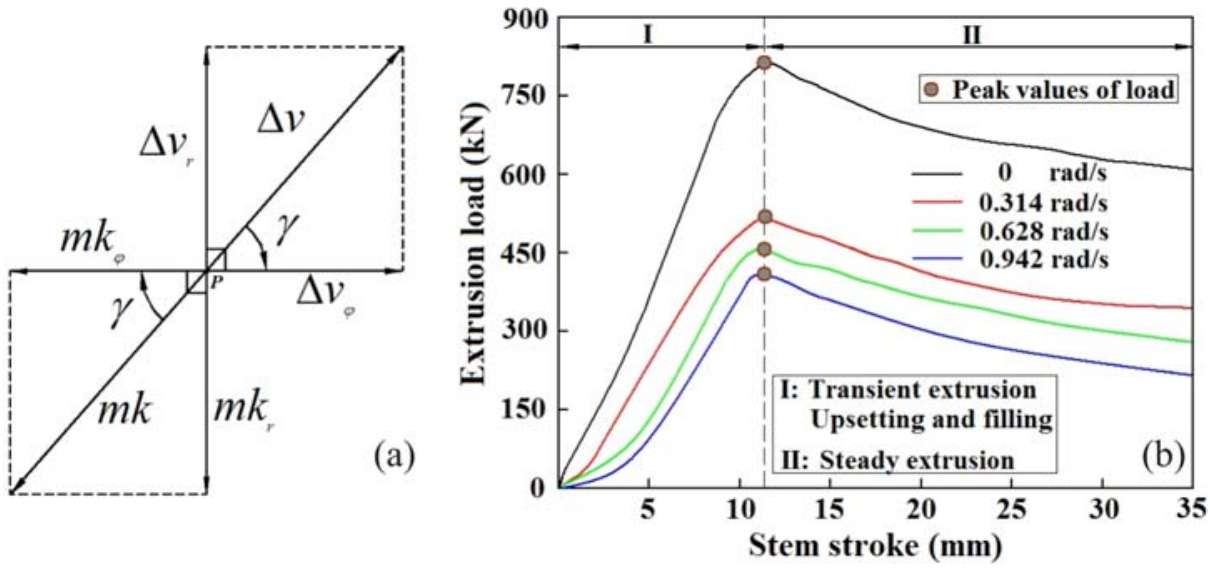

Fig. 7. Extrusion load comparison with different rotating speed: stress state of typical point (a), extrusion load (b).

different rotating speed is shown in Fig. 7.

With the increasing of stem stroke, the extrusion load presented decreasing tendency after increasing, although there was different rotating speed. From the values comparison, the peak value of extrusion load was $765 \mathrm{kN}$ for the conventional extrusion. The extrusion load decreased when the torque on the container was applied. When the rotating speed was $0.314 \mathrm{rad} \mathrm{s}^{-1}$, the peak value of extrusion load reached $510 \mathrm{kN}$. When rotating speed was doubled and tripled, 

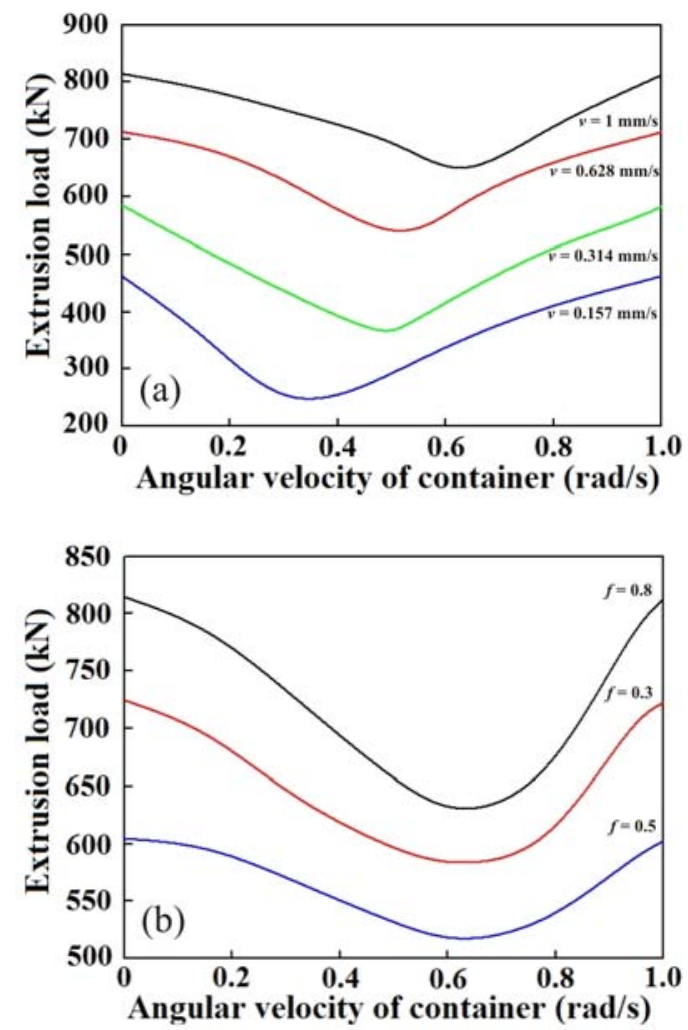

Fig. 8. Theoretical processing model: extrusion speed (a), friction factor (b).

the peak value of extrusion load decreased by 9 and $15 \%$, respectively. Comparing with conventional extrusion, the amplitude decrease reached $50 \%$. The research results indicated that applying torque on the container changed the direction of friction and the extrusion load could be decreased. The effect of load decreasing can be achieved by extrusion through rotating container.

The peak value of extrusion load is the critical factor for choosing a press in process design. The interaction among extrusion speed, rotating speed of container and friction factor is shown in Fig. 8.

The change rule among extrusion speed, rotating speed and extrusion load is shown in Fig. 8a. It can be seen from the figure, with the increasing of rotating speed, the peak value of extrusion load with different conditions of rotating speed presented a tendency to increase after decreasing and the peak value of extrusion load increased with the increasing of extrusion speed when the rotating speed was the same. The extrusion load curve moved from the low rotating speed area when the extrusion speed was $0.157 \mathrm{~mm} \mathrm{~s}^{-1}$ to high rotating speed area when the extrusion speed was $1 \mathrm{~mm} \mathrm{~s}^{-1}$. Due to the analysis above, the direction of friction was collectively determined by extrusion speed and rotating speed. When the extrusion speed was low, the axial component of friction could be

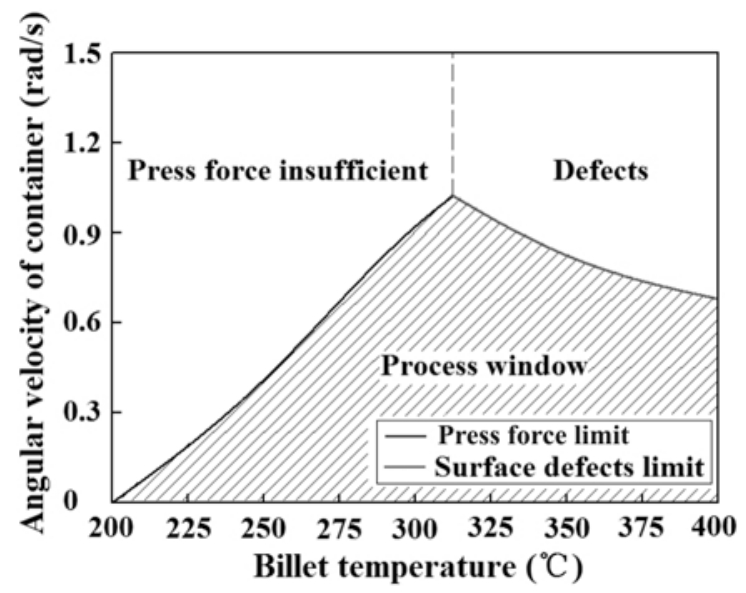

Fig. 9. Extrusion limit diagram.

decreased by applying a torque on the container, and the peak value of extrusion load obviously decreased. When the extrusion was high, slippage appeared when the rotating speed reached a certain degree.

For extrusion speed $1 \mathrm{~mm} \mathrm{~s}^{-1}$, the change rule among friction factor, rotating speed of container and peak value of extrusion load is shown in Fig. 8b. With the increase of rotating speed, the peak value of extrusion load increased after decreasing under the conditions of different extrusion speed. The peak value of extrusion increased with the increasing of friction factor and for the same rotating speed nearby $0.63 \mathrm{rad} \mathrm{s}^{-1}$.

The match of interaction between extrusion and rotating speed determined the change rule of the peak value.

\subsection{Forming limit}

The extrusion load is a function of the resistance to deformation. The characteristics of the flow stress decrease and plastic increase are useful in hot extrusion. But in the process of extrusion, the process could not be carried out when the extrusion load exceeded the rated load. An ultimate strength of alloy with the condition of multi-field coupling had a great influence on the quality and properties. For the extrusion speed $1 \mathrm{~mm} \mathrm{~s}^{-1}$ and friction factor 0.8 , the press limit curve and surface limit curve under the match condition of rotating speed and billet temperature are shown in Fig. 9.

It can be seen from Fig. 9, when retained other conditions, the extrusion limit curve presented a tendency of monotonous increase. Under the condition of rated load, with the increasing of billet temperature, the rotating speed can be increased synchronously. From the curve distribution of an alloy ultimate strength, the rotating speed required to be decreased so as to overcome the bad effect when the billet temperature 


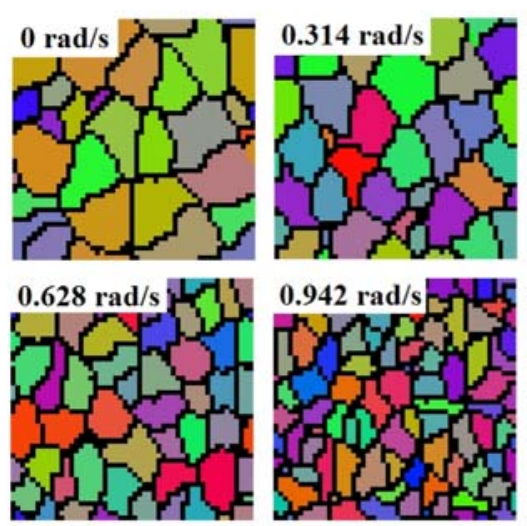

(a)

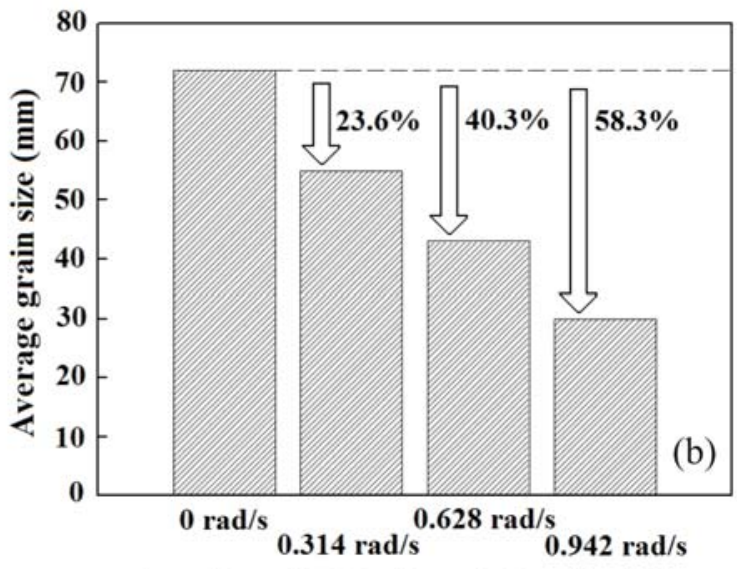

Angular velocity of container ( $\mathrm{rad} / \mathrm{s})$

Fig. 10. Average grain size comparison with different rotating speed: microstructure of grains (a), average grain size (b).

exceeded to a certain degree. These two limit curves divided the extrusion limit diagram into three parts. The rotating speed and billet temperature had direct influence on the process of extrusion. The part located in the left of press force limit presented a problem of press force insufficiency. And the part located in the right of surface defects limit presented hot cracking and grain boundary melt. The shadow area was reasonable process window. Rotating speed reached its maximum at the intersection point of these two limit curves.

\subsection{Microstructure}

Billet underwent shear deformation in the extrusion through rotating container, and this had an important effect on the microstructure. The microstructure comparison with different rotating speed is shown in Fig. 10.

It can be seen from Fig. 10 that the extrudate presents a refining trend with the increase of rotating speed. When rotating speed was $0.942 \mathrm{rad} \mathrm{s}^{-1}$, the microstructure showed that new grains appeared in the grain boundary. This phenomenon proved that dynamic recrystallization occurred. The average grain size comparison showed that when the rotating speed increased from 0 to $0.314 \mathrm{rad} \mathrm{s}^{-1}$, the average grain size decreased by $23.6 \%$. The grain size decreased by 40.3 and $58.3 \%$ when the rotating speed increased to 0.628 and $0.942 \mathrm{rad} \mathrm{s}^{-1}$. Therefore, a grain refinement can be realized by applying a torque on the container and increasing the rotating speed appropriately. The mechanical properties of extrudate improved.

\section{Conclusions}

1 . The flow velocity presented a specific vortex distribution in the extrusion through rotating container.
With the moving down of stem or rotating speed of container increasing the tendency of vortex flow became obvious. The difference of axial stress along the radial direction can be diminished by applying torque on the container. The uniformity of extrusion improved and the possibility of surface cracking was reduced.

2. Comparing with the conventional extrusion, the shapes of isothermal surfaces gradually changed to a shallow, even dis-shaped distribution from a hemispherical distribution. The temperature difference in the same section obviously diminished. The maximum temperature appeared decreased with the increasing of rotating speed.

3 . The results of different extrusion process indicated the match between extrusion speed and rotating velocity of container had an important effect on the peak value of extrusion load. With other conditions unchanged, an obvious effect could be obtained when extrusion speed was low and rotating velocity was high. But an excessive level of rotating velocity was not available when the extrusion speed was high, the load decreasing effect weakened when the slippage appeared.

4. According to the extrusion limit diagram of AZ31 magnesium alloy, surface defects can be caused through unreasonable match of process parameter. From the microstructure comparison, the grain refinement characteristic was obvious when rotating speed increased. Comparing with conventional extrusion, the average grain size decreased by $58.3 \%$, when the rotating speed was $0.942 \mathrm{rad} \mathrm{s}^{-1}$.

\section{Acknowledgements}

This paper was financially supported by the National Natural Science Foundation of China (No. 51205094). The authors would like to take this opportunity to express their sincere appreciation. 


\section{References}

[1] Li, F., Chu, G. N., Liu, E. L., Wu, R. Z., Zhang, X. L.: Part C: Journal of Mechanical Engineering Science, 12, 2011, p. 2927. doi:10.1177/0954406211411405

[2] Mizunuma, S., Iizuka, T., Mitsui, K., Okumura, H., Kohzu, M.: Steel Research International, 81, 2010, p. 1259.

[3] Mizunuma, S.: Materials Science Forum, 503-504, 2006, p. 185.

doi:10.4028/www.scientific.net/MSF.503-504.185

[4] Regguani, B., Donati, L., Tomesani, L.: International Journal of Material Forming, 3, Supplement 1, 2010, p. 395. doi:10.1007/s12289-010-0790-4

[5] Ceretti, E., Giardini, C., Giorleo, L.: International Journal of Material Forming, 3, Supplement 1, 2010, p. 323. doi:10.1007/s12289-010-0772-6

[6] Ketabchi, M., Mohammadi, H., Izadi, M.: Arabian Journal for Science and Engineering, 37, 2012, p. 2287. doi:10.1007/s13369-012-0320-4
[7] Atwell, D. L., Barnett, M. R.: Metallurgical and Materials Transactions A, 38, 2007, p. 3032. doi:10.1007/s11661-007-9323-2

[8] Davies, C., Barnett, M.: Journal of Minerals, Metal \& Materials Society, 5, 2004, p. 22. doi:10.1007/s11837-004-0121-7

[9] Lapovok, R. Y., Barnett, M. R., Davies, C. H. J.: Journal of Materials Processing Technology, 146, 2004, p. 408. doi:10.1016/i.jmatprotec.2003.12.003

[10] Li, L., Zhou, J., Duszczyk, J.: Journal of Materials Processing Technology, 172, 2006, p. 372. doi:10.1016/j.jmatprotec.2005.09.021

[11] Wang, L. L., Zhou, J., Duszczyk, J., Katgerman, L.: Tribology International, 56, 2012, p. 89. doi:10.1016/j.triboint.2012.01.012

[12] Wang, L. L., Yang, H. L.: Tribology International, 56, 2012, p. 99. doi:10.1016/j.triboint.2012.06.006 